\title{
Arterial Stiffness and Vascular Aging: From Pathophysiology to Treatment, with a Look at the Future
}

\author{
Agostino Virdis ${ }^{1}$ (D)
}

Received: 13 February 2018/Accepted: 16 February 2018/Published online: 22 February 2018

(C) Springer International Publishing AG, part of Springer Nature 2018

In the last decade, a growing literature raised arterial stiffness as a "hot topic" in terms of pathophysiology of cardiovascular (CV) system and clinical relevance [1, 2]. In order to comprehend the main aspects of such interest, a brief explanation of the "Windkessel" model is required. The arterial tree can be assimilated to a distensible tube terminating at the peripheral resistances, in order to explain the two main functions of the arteries: the conduit function (to deliver an adequate supply of blood to peripheral tissues) and the cushioning function (to buffer pressure oscillations due to intermittent ventricular ejection). The viscoelastic properties of large arteries guarantee that the pulsatile pressure and flow resulting from intermittent ventricular ejection are smoothed out, leading to the peripheral microcirculation to delivery oxygen and nutrients to the tissues. The velocity of pulse wave propagation is inversely related to the distensibility of the tube. At its end, the pulse wave is reflected and retrograde waves are generated, that, summated with the forward wave generated by ventricular ejection, constitute the effective blood pressure (BP) curve, contributing to the amplitude of pulse pressure and systolic BP. When the arterial stiffness increases, the pulse wave is transmitted more rapidly and, once reflected from the peripheral vasculature, returns to the heart during left ventricular contraction, resulting in a greater augmentation of the central aortic systolic BP [3].

This article is part of the topical collection on Vascular Aging and Arterial Stiffness.

Agostino Virdis

agostino.virdis@med.unipi.it

1 Department of Clinical and Experimental Medicine, University of Pisa, Via Roma, 67, 56100 Pisa, Italy
Ageing is undoubtedly the most powerful determinant of arterial stiffness [4], a phenomenon largely amplified and anticipated by the concomitant influence of clinical conditions such as hypertension, dyslipidaemia, smoking or diabetes mellitus, thus detecting the so-called early vascular aging. In addition to BP elevation, which per se represents a major determinant, other important mechanisms have been documented to conspire to the development the vascular stiffness, including low-grade vascular inflammation, renin-angiotensin-aldosterone system (RAAS) over-activation and oxidative stress [5-7].

A multitude of invasive and non-invasive methods measuring arterial stiffness has been described. The most used and validated techniques involve the assessment of the timing of pulse wave velocity (PWV) between the carotid and femoral arteries, which at present represents a recognized biomarker for risk stratification in $\mathrm{CV}$ disease prevention [3].

Few years ago, an expert consensus document on the measurement of aortic stiffness in daily practice established a threshold of $10 \mathrm{~m} / \mathrm{s}$ as a cut-off value for carotidfemoral PWV in hypertensive patients [8]. A large body of evidence supports the predictive value of arterial stiffness assessed by this technique. In particular, it is recognized that arterial stiffness is a strong and independent predictor of all-cause and CV mortality, fatal and non-fatal coronary events and fatal strokes [9, 10]. Several studies put in evidence the additive value of PWV above and beyond traditional risk factors, including SCORE and Framingham risk score [11, 12]. Accordingly, when arterial stiffness is measured, a substantial proportion of patients at intermediate risk could be reclassified into a higher or lower CV risk $[12,13]$. For these reasons, the European Society of Hypertension has accepted carotid-to-femoral PWV as a gold standard aortic stiffness measurement and a marker of 
asymptomatic organ damage to use in hypertension management [14].

For all these reasons, it is conceivable that the possibility to ameliorate arterial stiffness and slow down the vascular aging constitutes a mandatory target for therapy.

In this issue of High Blood Pressure and Cardiovascular Prevention, a review article by Neves and co-workers [15] mainly focuses on the role of the RAAS on arterial stiffness and central hemodynamic.

In the first part of this elegant and up-dated review, it is described the state of the art on the RAAS hyperactivity in the pathophysiology of arterial stiffness. An important aspect touched by the Authors concerns the strict crosstalk, documented in several convincing experimental and human studies, between angiotensin II and oxidative stress generation via inflammatory stimulation, a deleterious loop rapidly leading to endothelial dysfunction, vascular remodeling and atherosclerosis.

In the second part of the article, the impact of RAAS inhibitors on arterial stiffness is analysed and discussed. Indeed, available literature on the effects of ACE-inhibitors, AT-1 antagonists and the most recent direct renin inhibitor on vascular stiffness are critically commented. The real intriguing aspect of this review concerns the new RAAS components, which have been recently identified and documented to be able to add new functions to the classic axis and amplify our knowledge on this complex system. In particular, the authors focus their attention to the angiotensin converting enzyme-2 (ACE-2), Ang (1-7), alamandine and Mas receptors, which are considered part of a vasoprotective axis of the RAAS, counteracting the harmful effects of the Ang I/ACE/Ang II/AT1 receptor axis. It is obvious that this look at the future underscores the concept that the RAAS is still an actual and modern axis, which has been not yet explored in toto, thus encouraging future studies aiming to identity novel pharmacological strategies for attenuating arterial stiffness and the process of vascular aging.

\section{Funding None.}

\section{Compliance with Ethical Standards}

Conflict of interest The author declares that they have no conflict of interest.

\section{References}

1. Veerasamy M, Ford GA, Neely D, Bagnall A, MacGowan G, Das $\mathrm{R}$, et al. Association of aging, arterial stiffness, and cardiovascular disease: a review. Cardiol Rev. 2014;22(5):223-32.
2. Vlachopoulos C, Xaplanteris P, Aboyans V, Brodmann M, Cifkova R, Cosentino F, et al. The role of vascular biomarkers for primary and secondary prevention. A position paper from the European Society of Cardiology Working Group on peripheral circulation: Endorsed by the Association for Research into Arterial Structure and Physiology (ARTERY) Society. Atherosclerosis. 2015;241(2):507-32.

3. Laurent S, Cockcroft J, Van Bortel L, Boutouyrie P, Giannattasio C, Hayoz D, et al. Expert consensus document on arterial stiffness: methodological issues and clinical applications. Eur Heart J. 2006;27(21):2588-605.

4. Virdis A, Bruno RM, Neves MF, Bernini G, Taddei S, Ghiadoni L. Hypertension in the elderly: an evidence-based review. Curr Pharm Des. 2011;17(28):3020-31.

5. Lacolley P, Regnault V, Segers P, Laurent S. Vascular smooth muscle cells and arterial stiffening: relevance in development, aging, and disease. Physiol Rev. 2017;97(4):1555-617.

6. Marchesi C, Paradis P, Schiffrin EL. Role of the renin-angiotensin system in vascular inflammation. Trends Pharmacol Sci. 2008;29(7):367-74.

7. Harvey A, Montezano AC, Touyz RM. Vascular biology of ageing-Implications in hypertension. $\mathrm{J}$ Mol Cell Cardiol. 2015;83:112-21.

8. Van Bortel LM, Laurent S, Boutouyrie P, Chowienczyk P, Cruickshank JK, De Backer T, et al. Expert consensus document on the measurement of aortic stiffness in daily practice using carotid-femoral pulse wave velocity. J Hypertens. 2012;30(3):445-8.

9. Laurent S, Boutouyrie P, Asmar R, Gautier I, Laloux B, Guize L, et al. Aortic stiffness is an independent predictor of all-cause and cardiovascular mortality in hypertensive patients. Hypertension. 2001;37(5):1236-41.

10. Vlachopoulos C, Aznaouridis K, Stefanadis C. Prediction of cardiovascular events and all-cause mortality with arterial stiffness: a systematic review and meta-analysis. J Am Coll Cardiol. 2010;55(13):1318-27.

11. Sehestedt T, Jeppesen J, Hansen TW, Rasmussen S, Wachtell K, Ibsen $\mathrm{H}$, et al. Thresholds for pulse wave velocity, urine albumin creatinine ratio and left ventricular mass index using SCORE, Framingham and ESH/ESC risk charts. J Hypertens. 2012;30(10):1928-36.

12. Mattace-Raso FU, van der Cammen TJ, Hofman A, van Popele NM, Bos ML, Schalekamp MA, et al. Arterial stiffness and risk of coronary heart disease and stroke: the Rotterdam Study. Circulation. 2006;113(5):657-63.

13. Mitchell GF, Hwang SJ, Vasan RS, Larson MG, Pencina MJ, Hamburg NM, et al. Arterial stiffness and cardiovascular events: the Framingham Heart Study. Circulation. 2010;121(4):505-11.

14. Mancia G, Fagard R, Narkiewicz K, Redon J, Zanchetti A, Bohm $\mathrm{M}$, et al. $2013 \mathrm{ESH} / \mathrm{ESC}$ Guidelines for the management of arterial hypertension: the Task Force for the management of arterial hypertension of the European Society of Hypertension (ESH) and of the European Society of Cardiology (ESC). J Hypertens. 2013;31(7):1281-357.

15. Neves MF, Cunha AR, Rabello Cunha M, Altenburg Gismond R, Oigman W. The role of renin-angiotensin-aldosterone system and its new components in arterial stiffness and vascular aging. High Blood Press Cardiovasc Prev. 2018. https://doi.org/10.1007/ s40292-018-0252-5. 